\title{
Umbilical Cord Tissue Derived Mesenchymal Stem Cells Can Differentiate into Skin Cells
}

https://doi.org/10.1515/biol-2018-0065

Received September 8, 2018; accepted October 31, 2018

\begin{abstract}
Autologous skin grafts are used to treat severe burn wounds, however, the availability of adequate donor sites makes this option less practical. Recently, stem cells have been used successfully in tissue engineering and in regenerative medicine. The current study aims to differentiate umbilical cord tissue derived mesenchymal stem cells (CT-MSCs) into skin cells (fibroblasts and keratinocytes) for use to treat severe burn wounds. After isolation, MSCs were characterized and their growth characteristics were determined. The cells were induced to differentiate into fibroblasts and keratinocytes using respective induction medium. Results indicated that CT-MSCs were spindle shaped, plastic adherent and positive for CD29, CD44, CD73, CD90 markers. CT-MSCs also showed high proliferative potential as indicated by cumulative population doubling, doubling time and plating efficiency. The MSCs were successfully differentiated into fibroblast and keratinocytes as indicated by morphological changes and expression of lineage specific genes. We propose that these differentiated skin cells which are derived from CT-MSCs can thus be used for the development of bioengineered skin; however, further studies are required to evaluate the utility of these substitutes.
\end{abstract}

Keywords: mesenchymal stem cells, cord tissue, bioengineered skin, skin cells, keratinocytes, fibroblasts

\footnotetext{
*Corresponding author: Mahmood S Choudhery, Tissue Engineering and Regenerative Medicine Laboratory, Department of Biomedical Sciences, King Edward Medical University, Lahore, Pakistan, E-mail: ms20031@yahoo.com

Qandeel Fatima, Tissue Engineering and Regenerative Medicine Laboratory, Department of Biomedical Sciences, King Edward Medical University, Lahore, Pakistan

Nakhshab Choudhry, Department of Biochemistry, King Edward Medical University, Lahore, Pakistan
}

\section{Abbreviations}

MSCs: Mesenchymal Stem Cells

CT-MSCs: Cord tissue derived Mesenchymal Stem Cells

KGF: Keratinocyte growth factor

FGF: Fibroblast growth factor

PBS: Phosphate buffer saline

DMEM: Dulbecco's modified eagle medium

PE: Plating efficiency

cPDs: Cumulative population doublings

DT: Doubling time

\section{Introduction}

Skin wound healing requires restoration of both the dermal and epidermal layers of skin. Normally re-epithelization occurs by proliferation of keratinocytes that migrate from the wound edges. Similarly, fibroblast proliferation and release of growth factors restore dermis. In severe burn injuries, the normal repair process becomes defective. Autologous skin grafts is a treatment for severe burn wounds. However, problems such as the availability of adequate donor sites (for use as a graft) and chances of infection (due to additional injury) make this option less practical [1]. Recently, the use of adult stem cells has become a promising approach for the treatment of several diseases and disorders [2]. These cells are found in different adult and neonatal tissues. Stem cells possess two important characteristics, this is the ability of selfrenewal and the ability to differentiate into specific cell types under appropriate culture conditions. Mesenchymal stem cells (MSCs) are a type of adult stem cells that have remarkable differentiation potential and could be utilized for repair and regeneration of lost tissues [3].

The high proliferation, multi-lineage differentiation capacity, immunomodulatory and immunosuppressive properties have made MSCs promising therapeutic candidates [4-6]. The MSCs can be isolated from adult (such as bone marrow, adipose tissue, synovial membrane, teeth etc.) and neonatal sources (such as cord blood, cord tissue) [7-10]. The current study has focused on umbilical 
cord tissue derived MSCs (CT-MSCs) as human cord tissue is readily available. There are certain advantages in using cord tissue, firstly, a large number of MSCs can be harvested and secondly, its isolation poses no risk to donors. Finally, CT-MSCs are less immunogenic and can be expanded extensively in vitro [11-12].

In the current study, MSCs were isolated from human umbilicalcord tissue byan explantculturetechnique. These cells were characterized and their growth characteristics were determined. CT-MSCs were differentiated into fibroblasts and keratinocytes by culturing in the respective induction medium. Results show that CT-MSCs exhibited spindle shape morphology, plastic adherent growth and expression of CD29, CD44, CD73, CD90 markers. The CT-MSCs exhibited high proliferative potential as indicated by cumulative population doubling and plating efficiency. On the other hand, the differentiated fibroblast and keratinocyte exhibited a changed morphology and had expression of lineage specific genes. We show that CT-MSCs can be differentiated into both types of skin cells (i.e. keratinocytes and fibroblasts) that could be used in the future development of bioengineered skin. However, further studies will be required to evaluate the utility of such substitutes.

\section{Material And Methods}

\subsection{Collection and isolation of cord tissues}

Human umbilical cord tissues were collected following full-term cesarean births. Around 3-5 inches of cord tissue was transferred from the hospital to the cell processing facility under sterilized conditions. All samples were obtained after written consent from the donors. All procedures were performed according to the protocol approved by local Institution Review Board at the King Edward Medical University.

CT-MSCs were obtained using the explant culture technique as previously described [13]. Briefly, after washing with PBS (phosphate buffered saline), tissue pieces were minced into small pieces. Complete culture medium (Dulbecco's Modified Eagle Medium (DMEM) + 1\% non-essential amino acids $+1 \%$ penicillin/streptomycin solution) supplemented with $10 \%$ fetal bovine serum (FBS) was added and minced tissue pieces were incubated in culture flasks at $37^{\circ} \mathrm{C}$ with $5 \% \mathrm{CO}_{2}$ in humid conditions.

Informed consent: Informed consent has been obtained from all individuals included in this study
Ethical approval: The research related to human use has been complied with all the relevant national regulations, institutional policies and in accordance the tenets of the Helsinki Declaration, and has been approved by Institution Review Board at the King Edward Medical University.

\subsubsection{Plating efficiency (PE)}

At passage 1,40 cells $/ \mathrm{cm}^{2}$ were plated into a $25 \mathrm{~cm}^{2}$ culture flask containing complete medium and incubated at standard culture conditions for 2 weeks. After 2 weeks of culture, the medium was removed and cell colonies were fixed with absolute methanol and stained with $0.1 \%$ crystal violet dye. Cell colonies (with more than 30 cells) were counted under phase contrast microscope and PE was measured using the following formula [13]

PE: [Total number of colonies/ number of cells initially plated] x 100

\subsubsection{Number and time of population doublings}

To determine population doublings, initial cell number and number of cells harvested at each passage was recorded. The following formulae were used to calculate the cumulative population doublings and population doubling time [13]

$$
\begin{gathered}
\text { cPDs }=\log \mathrm{N} / \mathrm{No} \times 3.33 \\
\text { DT }=\mathrm{CT} / \mathrm{cPDs}
\end{gathered}
$$

Where, 'cPDs' represent cumulative population doublings, 'No' is the number of cells plated, ' $\mathrm{N}$ ' is the cells number harvested, 'CT' is the time in culture and 'DT' is the doubling time.

\subsection{Differentiation of CT-MSCs into skin cells}

For differentiation, CT-MSCs were cultured in keratinocyte or fibroblast induction medium for 2 weeks. The fibroblast differentiation medium contained DMEM (Gibco, Cat\#11995065), 10\% FBS (Merck, Cat\# 2020-07-31), 1\% penicillin/ streptomycin solution (Capricon, Cat\# CP13-1019), $5 \mathrm{ug} / \mathrm{ml}$ insulin (Sigma, Cat\# 19278-5ml) and $1 \mathrm{ng} / \mathrm{ml}$ basic fibroblast growth factor (Sigma, Cat\# F02901) while keratinocyte differentiation medium consisted of DMEM, 10\% FBS, 1\% penicillin/streptomycin solution, $0.5 \mathrm{mg} / \mathrm{ml}$ hydrocortisone (Sigma, Cat\# H-4001), 1\% insulin transferrin (Roche, Cat\# 13532600) and $15 \mathrm{ng} / \mathrm{ml}$ keratinocytes growth factor (Sigma, Cat\# H6666). Differentiation of CT-MSCs into fibroblasts and 
keratinocytes was confirmed by morphological changes and through polymerase chain reaction (PCR) using marker genes for fibroblast (desmin, collagen 3, vimentin, FGF7) and keratinocytes (CK1, CK10, CK14).

\subsection{Reverse transcription polymerase chain reaction (RT-PCR)}

Expression of lineage specific genes was carried out by RT-PCR. Briefly, cells were cultured for 15 days in the respective differentiation medium and total RNA was extracted using Trizol. Following extraction, the RNA was quantified using NanoDrop. For cDNA synthesis, 1.5ug of RNA sample was used using Wizscript cDNA synthesis kit. The WizPure ${ }^{\text {TM }}$ PCR master mix was used for quantitating the expression level of genes. Sequences for primer pairs and their product lengths (bp) are shown in Table 1. Gel bands were measured with image J software.

\subsection{Data Analysis Procedure}

Statistical analysis of data was performed using GraphPad Prism version 6. The data was expressed as mean \pm standard deviation. All experiments were performed on at least three samples in triplicate.

\section{Results}

\subsection{Isolation and characterization of MSCs}

We used an explant culture technique to obtain pure MSC population (Figure 1A). Within 7 days, MSCs appeared around the small pieces of cord tissue (Figure 1B). Cells grew rapidly around the tissue pieces (Figure 1C) and become confluent within two weeks (Figure 1D). CT-MSCs exhibited plastic adherent growth and spindle shaped morphology (Figure 1E). This spindle shaped morphology

Table 1: List of used primers and their sequences.

\begin{tabular}{|c|c|c|}
\hline Genetic Markers & 5'-3'sequenoe & Produd size \\
\hline Bet a Act in & $\begin{array}{l}\text { CGCATGGGTCAGAAGGATTC (F) } \\
\text { TAGAAGGTGTGGT GCCAGATT (R) }\end{array}$ & 137 \\
\hline $\operatorname{cD} 29$ & $\begin{array}{l}\text { GCAGTTGGTTT GCGATTAAG (F) } \\
\text { AAGGCATCACAGTCTTTCCA (R) }\end{array}$ & 233 \\
\hline $\mathrm{CD} 44$ & $\begin{array}{l}\text { A.GAAA,AAT GGTCGCTACA GCA (F) } \\
\text { CT GAAGT GCT GCTCCTTTCAC (R) }\end{array}$ & 571 \\
\hline $\mathrm{CD} 45$ & $\begin{array}{l}\text { CACT GCAGGGATGGATCTCA (F) } \\
\text { ACTCGTGGGTT CAGAACCTTCA (R) }\end{array}$ & 312 \\
\hline $\cos 3$ & $\begin{array}{l}\text { ACAACAGCCAACTGCTTTCAT (F) } \\
\text { TTCTCA,GCATTCCCGAA,AT (R) }\end{array}$ & 154 \\
\hline $\cos 90$ & $\begin{array}{l}\text { ATGAACCTGGCCATCAGCATGC (F) } \\
\text { CACGAGGTGTTCTGAGCAAGCA (R) }\end{array}$ & 344 \\
\hline $\mathrm{CK} 1$ & $\begin{array}{l}\text { GGAGGAGGAGGTGGTAGATTT (F) } \\
\text { GAGGTTGCTGATGTATGACT CG (R) }\end{array}$ & 388 \\
\hline CK10 & $\begin{array}{l}\text { GAGCAAGGAACTGACTACAG (F) } \\
\text { CT CGGTTTCAGCT GCAATCT (R) }\end{array}$ & 249 \\
\hline CK14 & $\begin{array}{l}\text { TGCTATTGGTGT CA GGGAAG (F) } \\
\text { GT GGCAAGGTT CTTTCTCC (R) }\end{array}$ & 277 \\
\hline Call स्द्रा-3 & $\begin{array}{l}\text { GTTGACCCTAACCAAGGATGCA (F) } \\
\text { GGAAGTT CAGGATT GCCGTAG (R) }\end{array}$ & 203 \\
\hline Vimentin & $\begin{array}{l}\text { CT GCGGGAGT AGTTGGAAAGT (F) } \\
\text { GGA,A,TT GGGACA,AA,ACATCCT (R) }\end{array}$ & 241 \\
\hline FGF 7 & $\begin{array}{l}\text { TGGTGAAGTTCATGGATGT CTATC (F) } \\
\text { CACAGGATGGCTT GAAGATGTA (R) }\end{array}$ & 212 \\
\hline Desmin & $\begin{array}{l}\text { CATCCTCAAGAAGGTGTTGGAG (F) } \\
\text { CA.AAGAGACGTGGGACGAGT (R) }\end{array}$ & 112 \\
\hline $40 c t$ & $\begin{array}{l}\text { GGCGTTCTCTTTGGAAAGGTGTTC (F) } \\
\text { CT CGAАСCAСАT CCTTCTCT (R) }\end{array}$ & 145 \\
\hline SSEA 4 & $\begin{array}{l}\text { CCGCGTCAAGAGGCCCATGAA (F) } \\
\text { CCCGCTT CT CGGT CTCGGACAA (R) }\end{array}$ & 148 \\
\hline
\end{tabular}


was maintained even in late passages (Figure 1F). In addition, CT-MSCs were positive for the expression of mesenchymal lineage markers CD29, CD44, CD73, CD90 while being negative for CD45 as determined by RT-PCR (Figure 1G).

\subsection{Growth Characteristics}

The growth characteristics of CT-MSCs were assessed by measuring plating efficiency, cumulative population doublings, and doubling time. Plating efficiency of
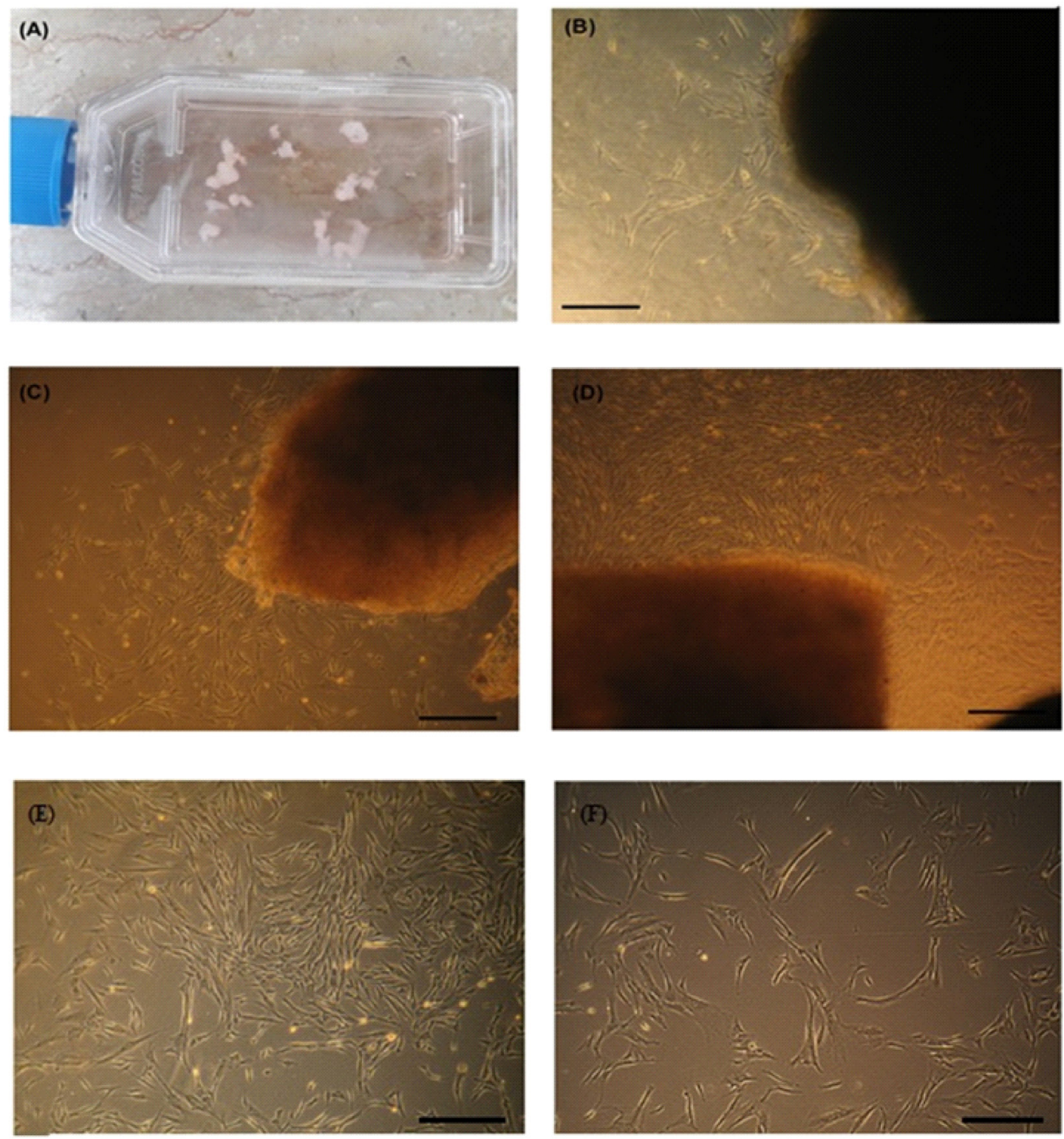

\section{(G)}

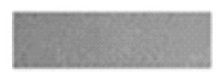

$\mathrm{CD} 45$
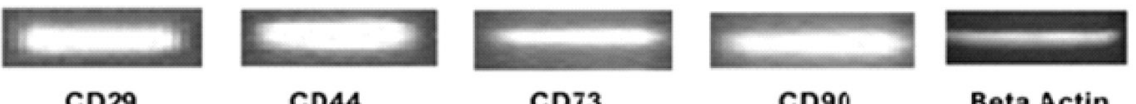

Beta Actin

Figure 1. Isolation and characterization of CT-MSCS: MSCs from cord tissue pieces were isolated using explant tissue culture (A). MSCS started to grow out of small cord tissue pieces within a week (B). Cells around cord tissue pieces after 10 days of culturing (C). Cells after 2 weeks of culture (D). CT-MSCs showed homogeneous spindle shaped morphology (E).CT-MSCs maintained their spindle shped morphology during passaging. The cells shown here are at passage 7 (F). PCR analysis showed positive expression of CD29, CD 44, CD73 and CD90, and negative expression of CD45 in CT-MSCS (G). 


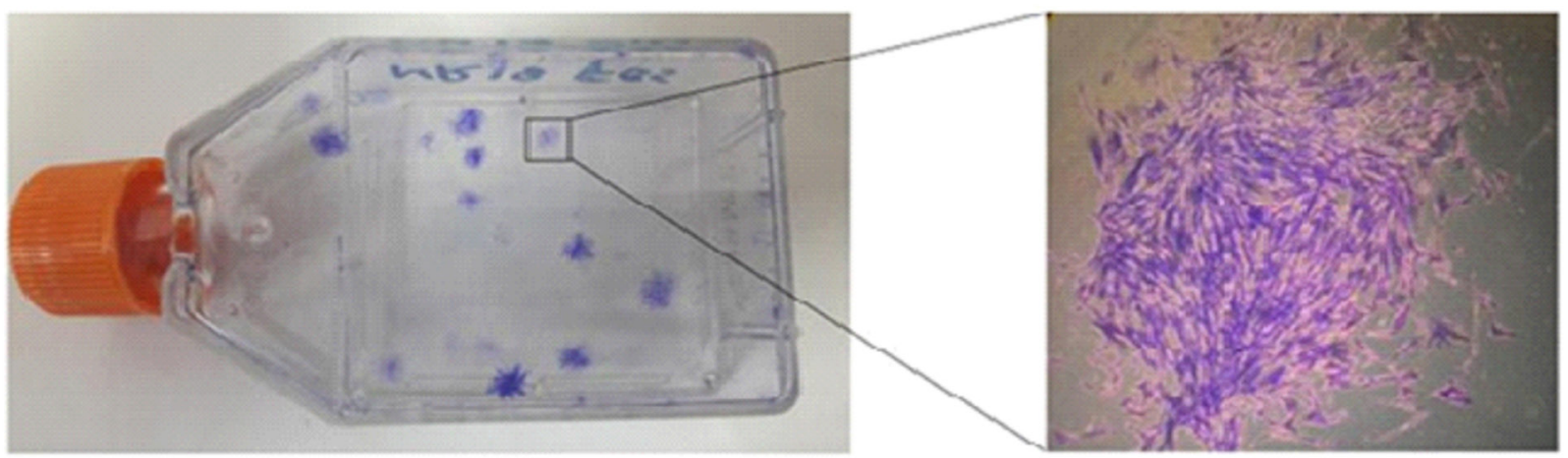

Figure 2. Plating efficiency of CT-MSCs. Inset shows a single colony of MSCs at high power (10X).

CT-MSCs was evaluated by culturing the cells at low numbers for the eventual growth into colonies. The number of colonies were counted after 2 weeks and this number was used to determine plating efficiency which was 4.225 \pm 1.7 (Figure 2).

To determine the number and time of population doublings at each passage, cells were counted with a hemocytometer and $10 \%$ cells were seeded into new culture flasks. The number of cumulative population doublings was $24.67 \pm 0.445$ for CT-MSCs. Similarly, the average population doubling time for CT-MSCs was $51 \pm 3$ hours.

\subsection{CT-MSCs can differentiate into keratino- cytes and fibroblasts}

CT-MSCs at passage $2(n=5)$ were differentiated into skin cells, either fibroblasts or keratinocytes. For differentiation into fibroblasts and keratinocytes, respective differentiation medium was used for 2 weeks. To serve as a control, cells were cultured in parallel in regular expansion medium.

Compared to the spindle shaped morphology of control group (Figure 3A), MSCs in the keratinocyte differentiated medium showed polygonal shape (Figure 3B). Similarly, RT-PCR results indicated that differentiated MSCs were positive for keratinocyte lineage markers (CK1, CK10, CK14) at day 7 and day 14 (Figure 3C, D). In contrast, the expression of OCT4 and SSEA4 stem cell markers was downregulated (Figure 3E, F).

Differentiation of CT-MSCs into fibroblasts was terminated after 14 days. Untreated CT-MSCs at 7 and 14 days were used as controls and showed no morphological changes (Figure 4A). This is compared to the prominent morphological changes when cultured in fibroblast induction medium (Figure 4B). The morphology of
CT-MSCs cultured in fibroblast induction medium changed considerably from spindle shape to a more elongated shape (Figure 4B). Furthermore, the expression of collagen-3, desmin, FGF-7, and vimentin was up-regulated after 14 days (Figure 4C, D) while the expression of SSEA4 and OCT4 was down-regulated (Figure 4E, F).

\section{Discussion}

Rapid repair and management of large skin ruptures is often necessary for the survival of patients. Minor wounds may heal without intervention, however, severe skin injuries require additional care. For severe burn injuries, autologous skin grafting is the best option available. However, there are certain limitations that make this treatment option less practical $[14,15]$. In the current study, CT-MSCs were differentiated into keratinocytes and fibroblasts with the future goal of using these to construct skin constructs. In the previous studies the MSCs isolated from different parts of placenta were either differentiated into fibroblasts or keratinocytes $[16,18]$. However, the current study is novel in that MSCs are isolated from umbilical cord tissue and we demonstrate successful differentiation into both types of skin cells (fibroblasts and keratinocytes). The isolation and subsequent differentiation of same cells into both types of cells is more convenient for the reconstruction of artificial skin substitutes. A caveat of our study is that we have not evaluated the utility of these differentiated skin cells in vitro or in vivo.

MSCs from the umbilical cord tissue were isolated by the explant culture method. This method is inexpensive and provided pure MSC population [13, 16]. We observed cell outgrowth within a week after the initial culture. Following 2-3 weeks of culture, a sufficient number of cells was obtained, which allowed for further experiments. The MSCs from tissue pieces exhibited plastic adherent growth, 


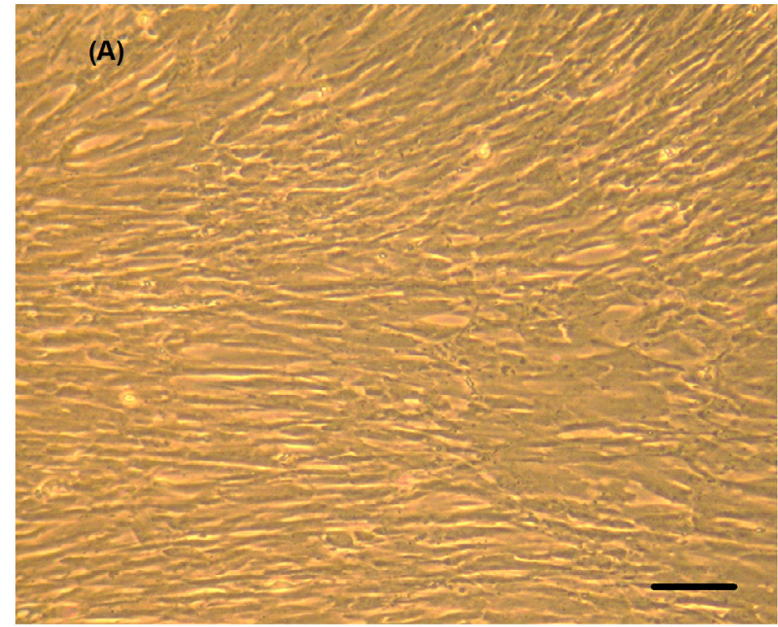

(C)

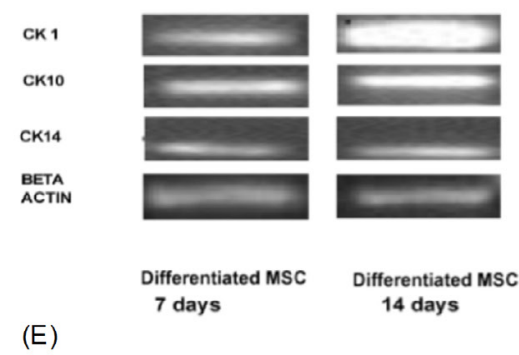

(E)

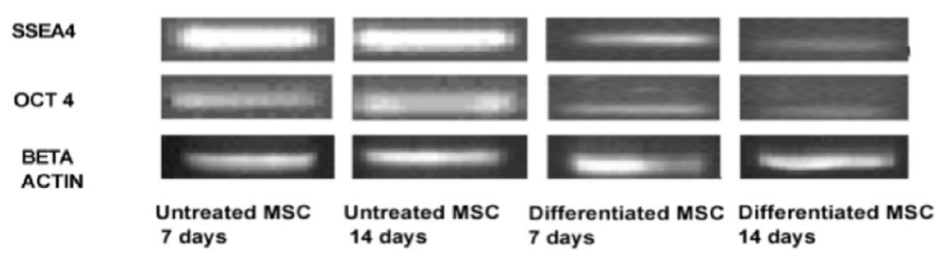

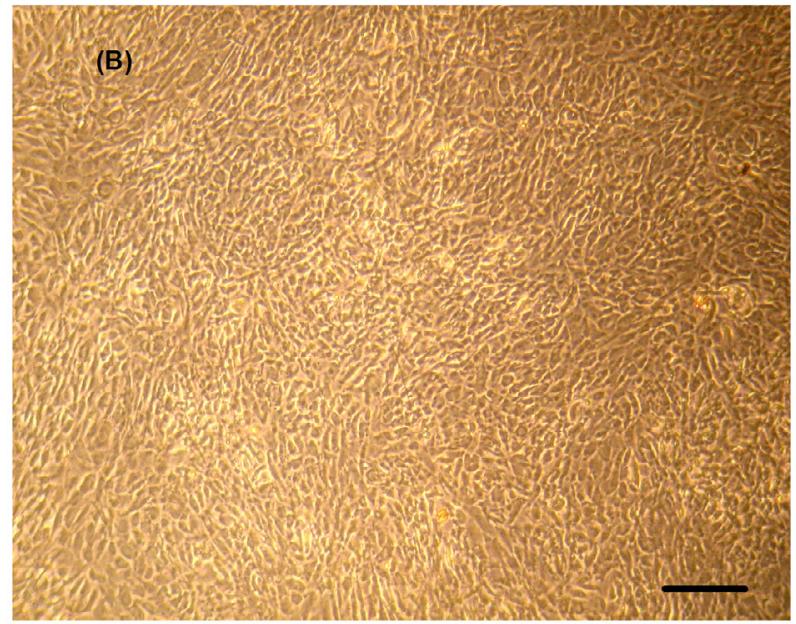

(D)

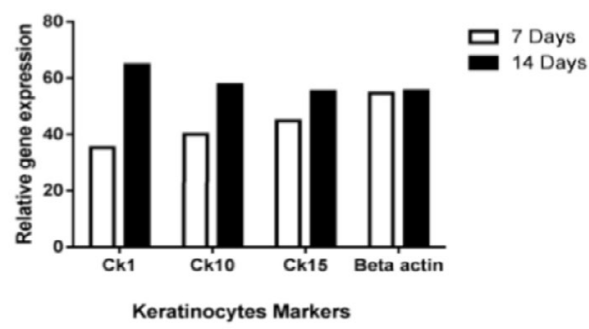

(F)

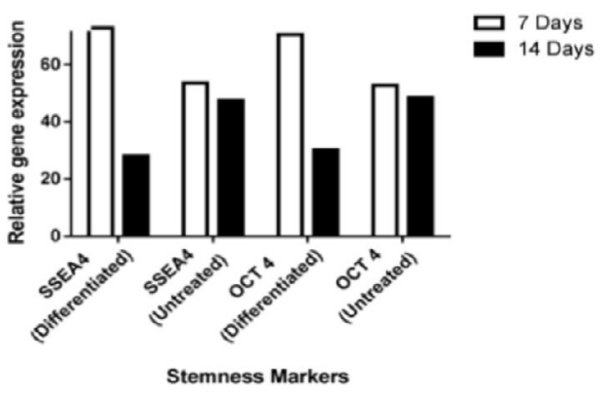

Figure 3. In vitro differentiation of CT-MSCs into keratinocytes: Untreated CT-MSCs exhibit spindle shape morphology (A). MSCs induced into keratinocytes exhibits a polygonal shape (B). Results of RT-PCR show that differentiated cells were positive for keratinocyte specific genes CK1, CK10 and CK14 (C). Quantification of gel bands using Imagel software (D). Expression of SSEA4 and OCT4 decreased during differentiation into keratinocytes $(\mathrm{E}, \mathrm{F})$.

spindle shaped morphology and positive expression of MSCs genes (CD29, CD44, CD73 CD90) and no expression of CD45 (a hematopoietic marker). These results were similar to already published reports [4, 10, 19-23]. CT-MSCs showed high proliferative potential as determined by plating efficiency and number of population doublings. The clonogenic potential of CT-MSCs was determined by plating efficiency amd was $4.225 \pm 1.7$. This was also similar to previous studies in the literature [24, 27]. The number population doublings of CT-MSCs was $24.67 \pm 0.445$ and their doubling time was $51 \pm 3$ hours. Similar results were reported by Choudhery and colleagues [13], and Mahmoud et al. [16].

To differentiate CT-MSCs into skin cells (fibroblasts and keratinocytes), they were cultured in respective differentiation medium for 2 weeks. In keratinocyte induction medium, differentiated CT-MSCs exhibited 

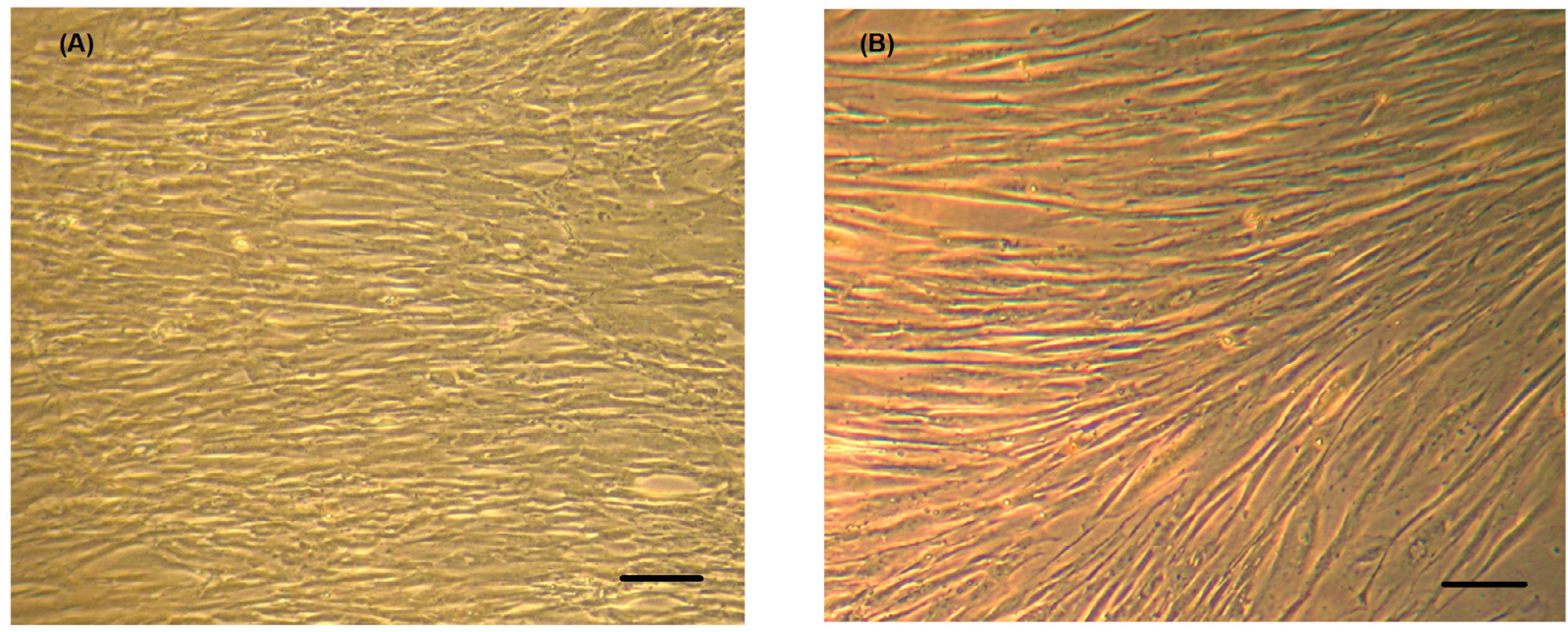

(C)

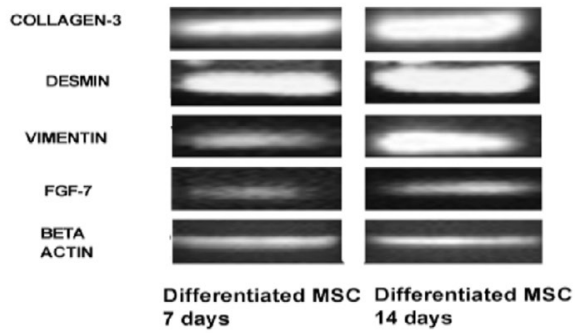

(E)

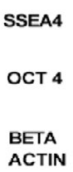

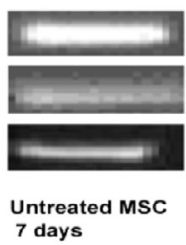

7 days
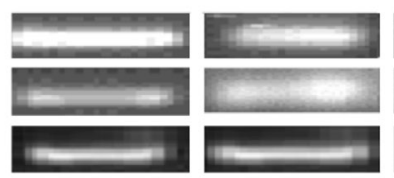

Untreated MSC 14 days
Differe
7 days

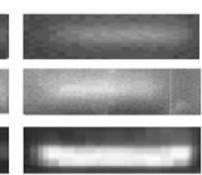
Differentiated MSC
14 days
(D)

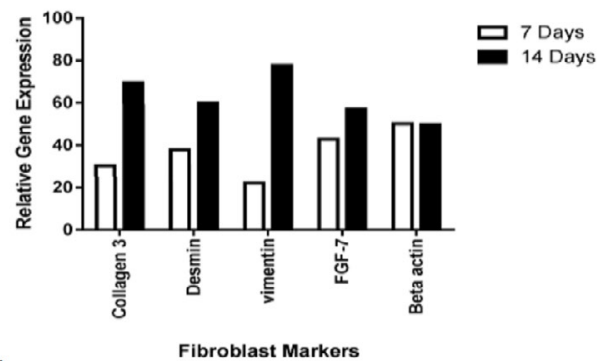

(F)

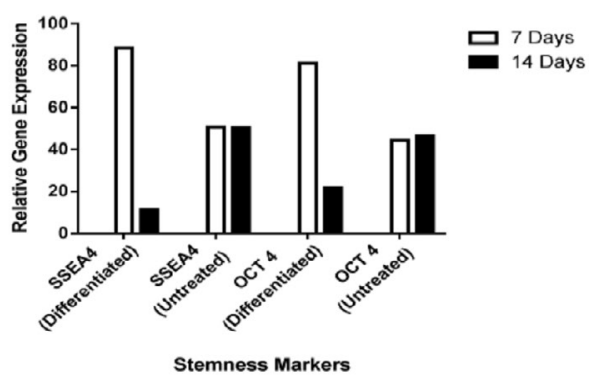

Figure 4. In vitro differentiation of CT-MSCs into Fibroblasts. Untreated CT-MSC exhibit spindle shape morphology (A). Treated CT- MSCS exhibited more elongated spindles morphology after culturing in fibroblast differentiation medium (B). Results of RT-PCR indicate that differentiated cells were positive for fibroblast specific genes (collagen-3, vimentin, FGF-7, desmin). RT-PCR analysis also showed upregulated expression of fibroblast specific markers, collagen-3, vimentin, FGF-7, and desmin after 14 days of differentiation versus 7 days (C). Quantification of gel bands with Imagel software (D). Expression of stem cell markers was downregulated at 14 days of culture in fibroblast differentiation media versus 7 days culture

polygonal morphology which was similar to keratinocytes [28].The differentiated cells were positive for the expression of CK1, CK10 and CK14 markers. Previous studies have demonstrated that keratinocytes express these markers [29- 30]. The CT-MSCs which were differentiated into fibroblasts with fibroblast differentiation medium, became more elongated when compared to the control.
Furthermore, these induced cells were positive for collagen-3, desmin, FSP-1 7 and vimentin which are fibroblast specific markers [31-33]. We also showed that during differentiation into skin cells (fibroblasts and keratinocytes), MSCs exhibited a progressive down regulation of pluripotency markers SSEA4 and OCT4 [3435]. In this study we demonstrate that MSCs with standard 
characteristics can be isolated from human placenta using the explant culture technique. These CT-MSCs can then be induced in vitro to differentiate into skin cells such as fibroblasts and keratinocytes. This successful differentiation was characterized by morphological changes and changes in gene expression profile. We propose that these cells may be used in the future to prepare an artificial skin substitute for use as a treatment for severe burn injuries.

Acknowledgments: We are thankful to the staff of the Lady Aichisen Hospital for providing cord tissue and cord blood samples. This work was supported by a grant from the King Edward Medical University, Lahore, Pakistan.

Conflict of interest: Authors state no conflict of interest

\section{References}

[1] Saaiq M, Zaib S, Ahmad S. Early excision and grafting versus delayed excision and grafting of deep thermal burns up to $40 \%$ total body surface area: a comparison of outcome. Annals of burns and fire disasters. 2012;25(3):143.

[2] Butler KL, Goverman J, Ma H, Fischman A, Yu YM, Bilodeau M, et al. Stem cells and burns: review and therapeutic implications. Journal of Burn Care \& Research. 2010;31(6):874-81.

[3] Birenboim R, Markus A, Goldstein RS. Simple generation of neurons from human embryonic stem cells using agarose multiwell dishes. Journal of neuroscience methods. 2013;14(1):9-14.

[4] Nombela-Arrieta C, Ritz J, Silberstein LE. The elusive nature and function of mesenchymal stem cells. Nature reviews Molecular cell biology. 2011;12(2):126-31.

[5] Jacobs SA, Roobrouck VD, Verfaillie CM, Van Gool SW. Immunological characteristics of human mesenchymal stem cells and multipotent adult progenitor cells. Immunology and cell biology. 2013;91(1):32-9.

[6] Caplan Al. Why are MSCs therapeutic? New data: new insight. The Journal of pathology. 2009;217(2):318-24.

[7] Rebelatto CK, Aguiar AM, Moretao MP, Senegaglia AC, Hansen P, Barchiki F, et al. Dissimilar differentiation of mesenchymal stem cells from bone marrow, umbilical cord blood, and adipose tissue. Experimental Biology and Medicine. 2008;233(7):901-13.

[8] Bianco P, Robey PG, Simmons PJ. Mesenchymal stem cells: revisiting history, concepts, and assays. Cell stem cell. 2008;2(4):313-9.

[9] Si YL, Zhao YL, Hao HJ, Fu XB, Han WD. MSCs: biological characteristics, clinical applications and their outstanding concerns. Ageing research reviews. 2011;10(1):93-103.

[10] Lu LL, Liu YJ, Yang SG, Zhao QJ, Wang X, Gong W, et al. Isolation and characterization of human umbilical cord mesenchymal stem cells with hematopoiesis-supportive function and other potentials. haematologica. 2006;91(8):1017-26.

[11] Weiss ML, Troyer DL. Stem cells in the umbilical cord. Stem Cell Reviews and Reports. 2006;2(2):155-62.
[12] Bongso A, Fong CY. The therapeutic potential, challenges and future clinical directions of stem cells from the Wharton's jelly of the human umbilical cord. Stem Cell Reviews and Reports. 2013;9(2):226-40.

[13] Choudhery MS, Badowski M, Muise A, Harris DT. Comparison of human mesenchymal stem cells derived from adipose and cord tissue. Cytotherapy. 2013;15(3):330-43.

[14] Li L, Xie T. Stem cell niche: structure and function. Annu. Rev. Cell Dev. Biol. 2005 Nov 10;21:605-31.

[15] Lorenti A. Wound healing: from epidermis culture to tissue engineering. CellBio. 2012;1(02):17.

[16] Mahmood R, Choudhery MS, Mehmood A, Khan SN, Riazuddin $S$. In vitro differentiation potential of human placenta derived cells into skin cells. Stem cells international. 2015;2015.

[17] Lee CH, Moioli EK, Mao JJ. Fibroblastic differentiation of human mesenchymal stem cells using connective tissue growth factor. InEngineering in Medicine and Biology Society, 2006. EMBS'06. 28th Annual International Conference of the IEEE 2006 Aug 30 (pp. 775-778). IEEE.

[18] El Ansary MA, Shaheen N, Farid R, Bishai IE. Mesenchymal Stem Cell Separation From Wharton's Jelly And Its Transdifferentiation Into Keratinocytes. Vox Sanguinis. 2012;103:253.

[19] Seshareddy K, Troyer D, Weiss ML. Method to isolate mesenchymal-like cells from Wharton's Jelly of umbilical cord. Methods in cell biology. 2008;86:101-19.

[20] Jo CH, Kim OS, Park EY, Kim B], Lee JH, Kang SB, et al. Fetal mesenchymal stem cells derived from human umbilical cord sustain primitive characteristics during extensive expansion. Cell and tissue research. 2008;334(3):423-33.

[21] Wegmeyer H, Bröske AM, Leddin M, Kuentzer K, Nisslbeck AK, Hupfeld J, et al. Mesenchymal stromal cell characteristics vary depending on their origin. Stem cells and development. 2013;22(19):2606-18.

[22] Dominici ML, Le Blanc K, Mueller I, Slaper-Cortenbach I, Marini FC, Krause DS, et al. Minimal criteria for defining multipotent mesenchymal stromal cells. The International Society for Cellular Therapy position statement. Cytotherapy. 2006;8(4):315-7.

[23] Zhang L, Chan C. Isolation and enrichment of rat mesenchymal stem cells (MSCs) and separation of single-colony derived MSCs. Journal of visualized experiments: JoVE. 2010(37).

[24] Kern S, Eichler H, Stoeve J, Klüter H, Bieback K. Comparative analysis of mesenchymal stem cells from bone marrow, umbilical cord blood, or adipose tissue. Stem cells. 2006;24(5):1294-301.

[25] Shetty P, Cooper K, Viswanathan C. Comparison of proliferative and multilineage differentiation potentials of cord matrix, cord blood, and bone marrow mesenchymal stem cells. Asian Journal of Transfusion Science. 2010;4(1):14.

[26] Jin HJ, Bae YK, Kim M, Kwon SJ, Jeon HB, Choi SJ, et al. Comparative analysis of human mesenchymal stem cells from bone marrow, adipose tissue, and umbilical cord blood as sources of cell therapy. International journal of molecular sciences. 2013;14(9):17986-8001.

[27] Corradetti B, Lange-Consiglio A, Barucca M, Cremonesi F, Bizzaro D. Size-sieved subpopulations of mesenchymal stem cells from intervascular and perivascular equine umbilical cord matrix. Cell proliferation. 2011;44(4):330-42.

[28] Fatimah SS, Tan GC, Chua K, Tan AE, Azurah AG, Hayati AR. Effects of keratinocyte growth factor on skin epithelial 
differentiation of human amnion epithelial cells. Burns. 2013;39(5):905-15.

[29] Laplante AF, Germain L, Auger FA, Moulin V. Mechanisms of wound reepithelialization: hints from a tissue-engineered reconstructed skin to long-standing questions. The FASEB Journal. 2001;15(13):2377-89.

[30] Sun HY, Zhou GM, Wang Q, Lin XC, Xu B. In vitro culture system for keratinocytes obtained from oral lichen planus lesions. Clinical oral investigations. 2014;18(4):1195-203.

[31] Yamada N, Uchinuma E, Kuroyanagi Y. Clinical trial of allogeneic cultured dermal substitutes for intractable skin ulcers of the lower leg. Journal of Artificial Organs. 2008;11(2):100-3.

[32] Dienus K, Bayat A, Gilmore BF, Seifert O. Increased expression of fibroblast activation protein-alpha in keloid fibroblasts: implications for development of a novel treatment option. Archives of dermatological research. 2010;302(10):725-31.
[33] Han Y, Chai J, Sun T, Li D, Tao R. Differentiation of human umbilical cord mesenchymal stem cells into dermal fibroblasts in vitro. Biochemical and biophysical research communications. 2011;413(4):561-5.

[34] Green H, Easley K, luchi S. Marker succession during the development of keratinocytes from cultured human embryonic stem cells. Proceedings of the National Academy of Sciences. 2003;100(26):15625-30.

[35] Noisa P, Ramasamy TS, Lamont FR, Jason SL, Sheldon MJ, Russell A, Jin X, Cui W. Identification and characterisation of the early differentiating cells in neural differentiation of human embryonic stem cells. PLoS One. 2012;7(5):e37129. 